\title{
Gerontology
}

\section{Dental Tissue Regeneration - A Mini-Review}

\author{
A.-H. Yen P.C. Yelick \\ Department of Oral and Maxillofacial Pathology, Division of Craniofacial and Molecular Genetics, Tufts University, \\ Boston, Mass., USA
}

\section{Key Words}

Teeth $\cdot$ Stem cells $\cdot$ Regeneration $\cdot$ Tissue engineering

\begin{abstract}
Background: With today's 21st century technological advancements, it is expected that individuals will either retain their natural teeth or obtain functional tooth replacements throughout their entire life. Modern dental therapies for the replacement of missing teeth largely utilize partial or complete dentures and titanium implants capped with prosthetic crowns. Although these prostheses serve a purpose, they are not equivalent, neither in function nor aesthetics, to natural teeth. Recent progress in dental tissue engineering has lent significant credibility to the concept that biological replacement teeth therapies may soon be available to replace missing teeth. Objective: In this review, we summarize the emerging concepts of whole-tooth replacement strategies, using postnatal dental stem cells (DSCs) and dental tissue engineering approaches. Methods: We provide a thorough and extensive review of the literature. Results: Current approaches to achieve clinically relevant biological replacement tooth therapies rely on the cultivation of DSCs capable of relaying odontogenic induction signals, through dental epithelial-mesenchymal cell interactions. DSC expansion and differentiation can be achieved by programming progenitor stem cells to adopt dental lineages, using instructive,
\end{abstract}

bioengineered scaffold materials. Periodontal ligament regeneration in particular has demonstrated significant progress recently, despite the somewhat unpredictable clinical outcomes, with regard to its capacity to augment conventional metallic dental implants and as an important component for whole-tooth tissue engineering. Following recent advances made in DSC and tissue engineering research, various research groups are in the midst of performing 'proof of principle' experiments for whole-tooth regeneration, with associated functional periodontal tissues. This mini-review focuses on recent and promising developments in the fields of pulp and periodontal tissue DSCs that are of particular relevance for dental tissue and whole-tooth regeneration. Conclusion: Continued advances in the derivation of useable DSC populations and optimally designed scaffold materials unequivocally support the feasibility of dental tissue and whole-tooth tissue engineering.

Copyright $\odot 2010$ S. Karger AG, Basel

\section{Introduction}

Tooth loss is an early indicator of accelerated aging. According to a recent study, persons who were edentulous at age 70 had a significantly higher risk of mortality 21 years later [1]. Although continental European and Scandinavian countries such as Sweden have exhibited

\section{KARGER}

Fax +41613061234

E-Mail karger@karger.ch

www.karger.com
(C) 2010 S. Karger AG, Basel

0304-324X/11/0571-0085\$38.00/0

Accessible online at:

www.karger.com/ger
Dr. Pamela C. Yelick

Department of Oral and Maxillofacial Pathology

Division of Craniofacial and Molecular Genetics, Tufts University

Boston, MA 02111 (USA)

Tel. +1 617636 2430, Fax +1 617636 2432, E-Mail pamela.yelick@tufts.edu 
reduced edentulism, from $51 \%$ in the $1901 / 1902$ birth cohort to $16 \%$ in the 1922 cohort [2], statistical predictions suggest that in the USA alone, of approximately 150 million adults, over 10 million new cases of edentulism will occur in the next decade. Edentulism not only results in reduced oral and social functions, but also remains a major public health issue [3].

Traditional methods to treat edentulism include complete denture therapy, which is associated with complications such as denture-induced stomatitis, soft tissue hyperplasia, traumatic ulcers, altered taste perception and burning mouth syndrome [1]. It is also well known that tooth loss leads to alveolar bone resorption [4]. Therefore, the need for alternative tooth replacement therapies is quite evident. The concept of osseointegration was introduced in the 1950s by Per-Ingvar Branemark, who observed the direct structural and functional bond formation between bone and titanium. Nowadays, endosseous implants are a commonly accepted treatment option. Titanium was subsequently regarded as a durable and biocompatible dental implant material which allowed the functional replacement of missing teeth. Since the 1950s, considerable improvements have been made to increase osseointegration of titanium implants, including surface modifications to improve the mechanical, physical and chemical characteristics of the implant [5]. Recently, biomimetic modification of the titanium implant surface has been proposed as a novel approach to further improve osseointegration [6]. However, osseointegration represents a direct connection between the implant and bone tissue and lacks the periodontium and cementum tissues present in naturally formed teeth, which function to cushion and modulate the mechanical stress of mastication. Therefore, strategies to generate dental implants with associated periodontal tissues have become a new approach in tooth replacement therapies [7].

A logical strategy for whole-tooth tissue engineering is to mimic natural tooth development, which is first evident as a localized thickening of the dental epithelium, the dental lamina, which subsequently invaginates into underlying neural crest-derived ectomesenchyme to form a bud. At the tooth initiation stage, odontogenic signals initiated in the inner dental epithelium stimulate proliferation and condensation of subjacent dental mesenchyme. Fibroblast growth factor-8, bone morphogenetic protein- 4 , Shh and Wnt10b, expressed by the dental epithelium, induce and regulate the expression of downstream transcription factors in the ectomesenchyme, including Barx1, Dlx1/2, Lhx6, Lhx7, Msx1, Pax9, Ptc and Lef1. Tooth shape specification occurs early in tooth de- velopment, at the dental lamina stage, and is regulated by several homeobox genes, such as Msxl, which are specifically expressed in the predental mesenchyme. The physical morphological process of tooth development begins at the cap stage and is coordinated by enamel knot signaling centers, transient structures present in the dental epithelium thought to be regulated by homeobox gene expression and which mark tooth cusp formation [8]. Secondary, tertiary and quaternary enamel knots sequentially appear and form the pattern of tooth crowns. By the late cap stage, the enamel knots will have disappeared by apoptosis.

Having received early odontogenic signals from the dental epithelium, the ectomesenchyme next becomes the principal source of signaling for subsequent tooth development, directing the dental epithelium to differentiate into enamel-secreting ameloblasts and the adjacent mesenchyme to differentiate into dentin-secreting odontoblasts [9]. At the bell stage, there is a recognizable tooth germ consisting of an organized enamel organ, dental papilla and dental follicle. The enamel organ, responsible for enamel formation, consists of inner and outer enamel epithelium, stratum intermedium and stellate reticulum. Ameloblasts, which are formed from the inner dental epithelium, differentiate to produce enamel, which is composed of more than $90 \%$ hydroxyapatite and is known to be the hardest tissue in the body. The cervical region of the inner and outer enamel epithelium gives rise to Hertwig's epithelial root sheath, a rudimentary tooth root structure that initiates radicular dentin formation and determines the root shape [10]. The dental papilla will give rise to pulp tissue, a complex, vital connective tissue composed of fibroblasts, blood vessels, nerves, lymphatic ducts and odontoblasts. Differentiated odontoblasts, cells derived from the mesenchymal cells directly subjacent to and induced by the inner enamel epithelium, are terminally differentiated, postmitotic cells that have withdrawn from the cell cycle and therefore cannot proliferate to replace irreversibly injured odontoblasts [11]. Functional odontoblasts exhibit a polarized columnar morphology that shifts into a resting state, becoming smaller and flatter after primary dentin formation. However, odontoblasts remain functional throughout their life and can produce secondary dentin in response to mild trauma. The dental follicle, a transient structure during tooth morphogenesis, eventually forms 3 major types of cells, i.e. cementoblasts, osteoblasts and fibroblasts [12]. Cementoblasts secrete cementum on the tooth root surface, while osteoblasts produce alveolar bone around the tooth roots. Collagen-producing fibroblasts give rise to peri- 

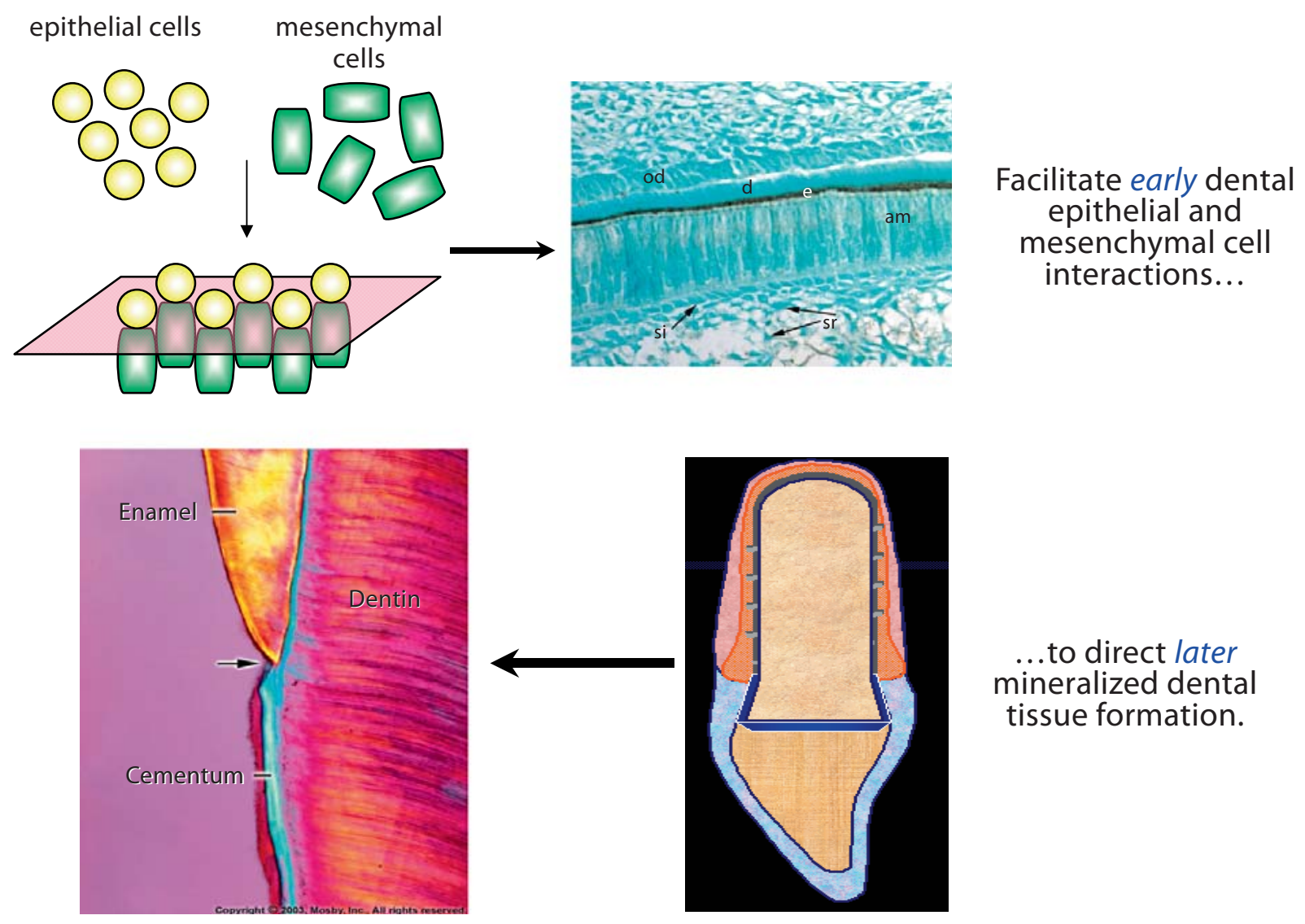

...to direct later mineralized dental tissue formation.

Fig. 1. Whole-tooth tissue engineering. od = Odontoblasts; $d=$ dentin; $e=$ enamel; $a m=$ ameloblasts; $s i=$ stratum intermedium; sr =stellate reticulum.

odontal ligament (PDL) tissues, which attach tooth roots to the surrounding alveolar bone through Sharpey's fibers extending into the cementum layer. The PDL functions as a mechanical cushion for masticatory forces, is an organ of proprioception via embedded nerve cells and is regarded as the main impetus for the tooth eruption process. The complex structure consisting of the PDL, adjacent cementum and surrounding alveolar bone is called the periodontium. After the eruption of a tooth into the oral cavity, the tooth is clinically divided into 2 parts, i.e. the crown and the root. Crowns are the visible structures in the oral cavity, and roots are the embedded tooth regions that connect the surrounding alveolar bone with the cementum, anchoring the tooth in place. Ana- tomically, the enamel-surfaced tooth extends to the cemento-enamel junction, while the cementum-covered tooth roots are present below the cemento-enamel junction. At the present time, there is extensive knowledge of tooth crown development, while relatively little is known about the molecular signaling mechanisms regulating tooth root development.

Based on these intricate and tightly regulated tooth developmental processes, as described above, dental tissue engineering approaches are currently being utilized to generate functional bioengineered replacement teeth and dental tissues that closely match the physical and mechanical properties of naturally formed tooth tissues, as schematized below (fig. 1). 


\section{Dental Stem Cells and Tooth Tissue Engineering}

Partial reparative tooth tissues had been created prior to the conception of tissue engineering [13]. For example, dentin production was shown to be induced by calcium hydroxide in pulp-capping procedures in the 1980s [14], although the underlying mechanisms remain elusive. Guided tissue regeneration was successfully used to regenerate periodontal tissues and had become a successful and widely available clinical therapy [15]. The emergence of whole-tooth tissue engineering stemmed from the demand for biologically based dental tissue and wholetooth replacements and was made possible by the marriage of biological, developmental and material sciences [13]. The concept underlying tissue engineering is to use ex vivo expanded progenitor cell populations, or stem cells, seeded into and grown within supporting biocompatible materials implanted in an appropriate environment, to create functional tissue replacements.

Stem cells are defined as clonogenic cells exhibiting the capacity for self-renewal and multilineage differentiation. Stem cells can be divided into 2 main types, i.e. embryonic stem cells (ESCs) and adult stem cells. Pluripotent ESCs are derived from the inner cell mass of mammalian blastocysts and can be maintained indefinitely in culture [16]. The demonstrated conversion of mouse ESCs into differentiated neurons in an animal model of Parkinson's disease, and into islet cells in diabetes, together with successful isolation and characterization of human ESCs, has stimulated vigorous interest in their potential use in clinically relevant applications in humans. Human ESCs promise a renewable source of progenitor cells that can be induced to differentiate into precursors of virtually any cell type. However, enthusiasm regarding ESC use is dampened by concerns regarding possible tumorigenic and malignant properties potentially exhibited by undifferentiated ESCs when implanted into ectopic sites, along with polarizing ethical issues regarding the use of human ESCs.

Until very recently it was generally assumed that stem cells in adult tissues were limited to specific cell fates. However, adult stem cells harvested from bone marrow [17], hematopoietic [18], neuronal [19] and mesenchymal tissues [20] have been demonstrated to differentiate into cell types that are derived from multiple germ layers, a feature defined as plasticity. The plasticity of adult stem cells remains a controversial issue, due to the current lack of adequate numbers of definitive differentiation markers, as well as a lack of reliably reproducible protocols and results. However, adult somatic cells infected with retro- viral or lentiviral vectors expressing the transcription factors Oct 4 and Sox2, along with either Klf4 and c-Myc, or Nanog and Lin-28, exhibit an ESC-like phenotype and have been named induced pluripotent stem (iPS) cells [21-24]. iPS cells behave similarly to ESCs, exhibiting the capacity to differentiate into virtually any cell type, potentially providing unlimited opportunities for tissue regeneration, while avoiding ethical issues surrounding the use of human embryos. Although issues remain regarding the possible tumorigenic potential of retrovirally transfected cells, improved viral-free transfection methods are currently being investigated to eliminate this concern [25]. Thus, the creation of iPS cells presents potentially tremendous opportunities for the development of patient-specific therapies.

\section{Dental Pulp Stem Cells}

The ability of human teeth to form reparative dentin in response to deep caries and mild trauma suggests that progenitor cells present in fully developed tooth pulp retain the ability to form functional odontoblasts, which can produce dentin-like hard tissues [26]. The theory is that undifferentiated mesenchymal progenitor cells existing in the dental pulp have the ability to differentiate into odontoblast-like cells and form new dentin in response to dental injury [27]. To better understand tooth regeneration capabilities, several populations of dental stem cells (DSCs) have been identified and characterized. Human dental pulp cells, derived from developing third molars and cultured in mineralization-enhancing conditions, were demonstrated to form odontoblast-like cells that produce dentin and also express the neuronal marker nestin [27]. Similar studies showed that human dental pulp stem cells (DPSCs) $[28,29]$ and stem cells harvested from human exfoliated deciduous teeth (SHED) [30] could be derived from adult and deciduous dental pulp, respectively, as indicated by their high proliferation and colony-forming ability in culture. Besides exhibiting the capacity to form mineralized tissues, DPSCs and SHED can express neural markers and also have the potential to differentiate into adipocytes [30]. When DPSCs were implanted subcutaneously into immunocompromised mice, dentin-pulp-like complexes formed, but lamellar bone did not. Dentin and bone formation, but not dentin-pulp complexes or surrounding alveolar bone, was observed in transplanted SHED in vivo. Although DPSCs and SHED appear to contain stem cells, they are also likely to contain heterogeneous populations of differentiating pulp cells, which further complicates analyses of these cells. 
Although it has been claimed that true mesenchymal stem cells (MSCs) could be identified using a collection of poorly defined markers [31], the resulting sorted cell population still exhibits varying degrees of multipotentiality, indicative of heterogeneity. The fact that clonal cell lines with a similar potential to regenerate bone in vivo exhibit nonidentical marker expression profiles [32] is indicative of the fact that cultured DSC populations change in culture, further confounding these studies. The task of isolating purified dental MSC populations faces similar difficulties to those encountered in generating purified populations of other postnatal stem cell types, which also lack suitable specific stem cell markers. However, the advantage with teeth is that they are one of the most accessible organs from which to derive stem cells, and DPSCs can be cryopreserved while retaining their multipotential differentiation capacity [33]. Therefore, dental MSCs can likely provide an autologous population of neural crest cell-derived stem cells for clinically relevant therapeutic purposes in humans.

Another unique population of stem cells isolated from human teeth is found at the tooth root apex. These cells are called DSCs of the apical papilla (SCAP) and have been demonstrated to differentiate into both odontoblasts and adipocytes. The higher proliferative potential of SCAP as compared with DPSCs makes this population of cells suitable for cell-based regeneration and preferentially for forming roots $[34,35]$.

\section{Periodontal Ligament Stem Cells}

In common with dental pulp, periodontal ligament (PDL) retains limited capacity to regenerate in response to mild trauma. Multipotent progenitors from human PDL have been identified and validated using methods similar to those used to characterize DPSCs/SHED, including single-colony selection and magnetic activated cell sorting with STRO-1, and PDL stem cells (PDLSCs) have been characterized as STRO-1 and CD146/MUC18 positive [36]. Human PDLSCs display cell surface marker characteristics and differentiation potential similar to bone marrow stromal stem cells and DPSCs. Under defined culture conditions, PDLSCs are multipotent and exhibit the ability to differentiate into cementoblast-like cells, adipocytes and fibroblasts [37]. After PDLSCs were transplanted into immunocompromised mice, cementum/PDL-like structures were formed. Human PDLSCs expanded ex vivo and seeded in 3-dimensional scaffolds (fibrin sponge, bovine-derived substitutes) were shown to generate bone [38]. These cells have also been shown to retain stem cell properties and tissue regeneration capac- ity even after recovery from solid-frozen human primary tissue [39]. These findings suggest that cryopreserved PDLSCs from extracted teeth could prove useful for clinically relevant therapeutic applications in the future.

\section{Dental Follicle Stem Cells}

The dental follicle has long been considered a multipotent tissue, based on its ability to generate cementum, bone and PDL from the ectomesenchyme-derived fibrous tissue. Bovine dental follicle cells exhibit the ability to form cementoblasts when transplanted into immunodeficient mice. Human dental follicle progenitor cells obtained from human third molars exhibit a characteristic ability to attach to tissue culture plastic, express differentiated tissue makers, including nestin and Notch-1, and to differentiate into PDL-like structures, including bone and cementum [36-40]. Further analysis revealed the heterogeneous nature of cell populations in developing dental follicles, through analysis of mineralization capacities in vitro and characterization of growth factor and matrix protein gene expression patterns from several clonally derived dental follicle cell lines cultured using identical conditions. DSCs were identified in the dental follicles of molars by Hoechst staining, alkaline phosphatase staining, the expression of side population stem cell markers and the demonstrated ability to differentiate into not only osteoblasts/cementoblasts but also adipocytes and neurons.

\section{Dental Epithelial Stem Cells}

Oral ectoderm-derived ameloblasts are unable to proliferate or regenerate once they have reached the maturation stage of development. Continuously growing mouse incisors, and molars in some mammalian species, exhibit constantly replenishing populations of enamel organ tissue-derived stellate reticulum, stratum intermedium and surrounding outer enamel epithelial cells, providing a source of tissues to harvest for characterization of dental epithelial stem cells and analyses of dental epithelial tissue.

Continuously erupting mouse incisors exhibit an epithelial DSC niche located at their labial apical end, known as the cervical loop, located at the junction of the inner enamel epithelium and the outer enamel epithelium at the apex of the enamel organ. The cervical loop is considered to be a determinative region regulating odontogenesis, based on its ability to produce both enamel and dentin. Early morphological observations revealed that most mitotic cells were located in the inner enamel epithelium and stratum intermediate, while the stellate reticulum 
and outer enamel epithelium exhibited relatively reduced proliferative activity. Recently, a specialized structure located at the apical region of the labial cervical loop in mouse incisors was characterized and named the 'apical bud' [41]. Apical buds were suggested to act as epithelial DSC-containing compartments that could differentiate into ameloblasts through interaction with adjacent mesenchymal cells, including DPSCs.

\section{Periodontal Tissue Regeneration}

Periodontal tissue regeneration represents the ultimate goal of periodontal therapy and entails the formation of all components of the periodontium, including gingiva, PDL, cementum and alveolar bone. In the early 1980 s, attempts to regenerate periodontal tissues largely focused on therapies designed to demineralize tooth root cementum tissue in order to expose underlying collagen fibers, with which newly formed collagen fibers could subsequently integrate. This procedure often caused ankylosis and tooth root resorption, however, and thus the procedure failed [42]. Another approach to periodontal regeneration was the introduction of bone grafts into the periodontal defect site. Although utilization of such grafting materials for clinical repair of periodontal defects resulted in increased attachment levels of periodontal tissues and radiographic evidence of bone fill, histological assessment usually revealed that these materials had little osteoinductive capacity and generally appeared to be surrounded by dense fibrous connective tissue rather than organized periodontal tissue [42]. In recent years, guided tissue regeneration has become the gold-standard surgery for periodontal tissue regeneration. This procedure involves draping a biocompatible membrane over the periodontal defect from the root surface to the adjacent alveolar bone, often in combination with a bone graft. The barrier membrane prevents unwanted epithelium and gingival connective tissue from entering the healing site, while promoting repopulation of the defect site by cells migrating in from the PDL [38]. The rather limited success of this approach has led scientists to develop methods to improve this therapy, through the addition of exogenous growth factors and via stem cell therapy.

Commonly used growth factors for PDL regeneration therapies include bone morphogenetic proteins, plateletderived growth factor, Emdogain and recombinant amelogenin protein. The resultant improved regenerative capability could be related to increased recruitment of progenitor MSCs, which subsequently differentiate to form PDL tissue. Recently, PDLSCs transfected with expression vectors for platelet-derived growth factor and bone morphogenetic protein were investigated in periodontal tissue engineering models $[43,44]$. These studies revealed the regeneration of normal periodontal tissues, containing organized cementum, alveolar bone and the PDL attachment apparatus. The possibility of constructing a root-periodontal tissue complex was further successfully demonstrated using a pelleted hydroxyapatite/ tricalcium phosphate scaffold containing SCAP, coated with PDLSC-seeded Gelfoam, implanted and grown in the minipig tooth socket [30].

The multipotent differentiation properties of PDLSCs for generating both hard and soft tissues were further demonstrated by constructing multilayered cell sheets supported by woven polyglycolic acid. Transplanted cellseeded polyglycolic acid sheets regenerated new bone, cementum and well-oriented collagen fibers when inserted into root surfaces. In addition to PDL-derived DSCs, bone marrow-derived MSCs and adipose-derived stem cells have been shown to promote periodontal tissue regeneration as well, although the mechanisms regulating these processes have not been elucidated $[44,45]$. One hypothesis is that controlled MSC differentiation could be developed as a therapeutically relevant approach for PDL tissue regeneration. The combined use of transplanted MSCs and added exogenous signaling molecules could accelerate the directed differentiation of MSCs in vivo, providing more effective promotion of periodontal tissue regeneration.

Successful therapies for PDL tissue regeneration will not only facilitate the treatment of periodontal diseases, but may also be used to improve current dental implant therapies. Numerous attempts to reconstruct periodontal tissues around dental implants revealed the challenge of avoiding fibrous tissue encapsulation and the formation of functional cementum on the implant surface [7].

\section{Whole-Tooth Regeneration}

Whole-tooth regeneration efforts largely consist of two approaches: one involves in vivo implantation of immature tooth structures grown in vitro from dental progenitor cells, while the other uses in vitro expanded, cultured dental progenitor cell populations seeded onto polymer scaffolds and implanted in vivo. Significant progress in adult stem cell biology, biomaterials science and the identification and characterization of DSCs have 
contributed to recent successes in regenerating tooth germs.

Early studies showed that it was possible to regenerate tooth crowns from intact or partially dissected tooth germs if suitable environments were provided, such as in vitro organ culture, in vivo grafting on chick chorioallantoic membrane, ocular or subrenal grafts or subcutaneous transplants [46-48]. Each of these implant sites provides nutrients and oxygen to nurture tooth germ differentiation into mature teeth. Thus, there are several choices for cultivating small tooth primordia prior to implantation into their anatomically relevant site in the jaw. Optimally, the implant setting should reproduce an appropriate 3-dimensional organization for transplanted cells to support functional differentiation, while avoiding xenograft rejection. Therefore, organotypic culture is arguably the most relevant model system in which to grow teeth in vitro.

By applying traditional tissue engineering methods, tooth-like structures can be produced from biodegradable polymer scaffolds seeded with dissociated tooth germ cells obtained from postnatal pigs or cultured rat tooth bud cells grown in the omentum of immunocompromised mice [49]. Although harvested implants contained anatomically correct tooth crowns consisting of organized enamel, dentin and pulp tissues closely resembling that of naturally formed teeth, they also contained disorganized dental tissue and furthermore did not adopt the size or shape of the biodegradable scaffold. Despite these shortcomings, these results confirmed the ability of mixed, heterogeneous populations of dental epithelial and mesenchymal cells derived from dissociated adult tooth germs to reaggregate within biodegradable scaffolds, interact and recapitulate odontogenic differentiation, to form adult tooth structures.

Examining the autonomous reaggregation capacity of dissociated tooth germ cells further disclosed that cap stage dental mesenchyme could induce dental epithelial cell histogenesis even when positional memories have been lost. Tooth morphogenesis of reaggregated tooth germ cells showed characteristic developmental features including the formation of functional odontoblasts and ameloblasts, pulp and cusp formation and, following longer culture times, the formation of tooth roots and PDL tissues [50]. These experiments demonstrated that cap stage dental mesenchyme could control tooth cusp number and further reinforced the demonstrated ability for mesenchymal and dental epithelium reaggregation.

Recently, it was found that dental cell-seeded tooth scaffold constructs grown in a coculture system appeared to result in an improved ability to control tooth size and shape. Dental mesenchymal cell-seeded polyglycolic acid mesh draped with dental epithelial cell-seeded collagen sponge gels that allowed for direct contact between dental epithelial and mesenchymal cells were used to generate organized tooth structures derived from dissociated canine and porcine molar tooth germs [51, 52]. In a similar study, organized dentin-pulp complex, cementum and PDL were shown to form in approximately one third of experimental implants generated using gelatin-chondroitin-hyaluronan-tri-copolymer [53].

Although whole-tooth in vitro cell culture methods are being further developed and improved, the task remains to identify available DSC populations to replace the use of embryonic dental epithelial and mesenchymal cells. The search for alternative sources of dental epithelium and mesenchyme is encouraged by the fact that dental epithelium can be created from non-teeth-bearing tissues and that non-dental, neural crest-cell derived mesenchyme can become competent for odontogenesis when allowed to interact with inductive dental epithelium. These experiments established the feasibility of instructing non-dental tissues to develop into teeth. Presumptive DSCs such as DPSCs have been used to regenerate partial teeth structures, but not an entire, functional biological tooth. Due to the limited in vitro expansion abilities of DPSCs, SHED and other DSC populations, significant efforts have been made to establish transgenic DSC lines expressing transgenes, including human telomerase reverse transcriptase, SV40 T antigen and human papillomavirus genes, or through the identification of spontaneously immortalized dental follicle cells, Hertwig's epithelial root sheath, cementoblasts, dental papilla, PDL, cervical loop epithelium, ameloblast and odontoblast lineage cells. One of the major technical advantages of this approach is that cells can be produced, characterized and controlled relatively easily without the need to derive material repeatedly from primary tissue. These lines could therefore potentially be used to generate dental structures in vivo.

A bigger challenge is to identify non-DSC populations as potential replacements for dental epithelium and mesenchyme. It has been demonstrated that ESCs, neural stem cells and bone marrow-derived cells can respond to inductive signals from dental epithelium and express odontogenic genes, and that bone marrow stromal cells and dental epithelial recombinant explants can in fact form tooth crowns composed of organized enamel, dentin, pulp and surrounding bone [54]. The teeth produced were of the appropriate size and shape for mouse molars, 
reinforcing the idea that odontogenic signals could instruct tooth crown formation in embryonic or adult stem cells without the use of a scaffold. Additional studies using c-kit-enriched bone marrow stromal cells demonstrated their ability to differentiate into ameloblast-like cells [55], suggesting their potential to differentiate into both dental epithelial and dental mesenchyme-derived tissues.

\section{Current Challenges in Dental Tissue Engineering}

A sufficient number of cell sources currently exist for use in generating bioengineered dental mesenchymal tissue-derived tissues, including DPSCs, SHED, SCAP and PDLSCs. In contrast, human dental epithelial stem cell sources are limited for the following two reasons. Firstly, dental epithelial cells undergo apoptosis after enamel formation is completed and therefore are no longer present in erupted teeth. Undifferentiated wisdom or impacted teeth are thus the only available sources of human dental epithelium and are obtained either from children or young adults. Secondly, ex vivo dental epithelial expansion can be difficult, due to the fact that it is inherently more difficult to expand epithelial cells in culture as compared to mesenchymal cells. Therefore, in order to routinely bioengineer human whole teeth containing functional enamel, alternative dental epithelial cell sources will have to be identified. Potential solutions to this problem are provided by studies demonstrating that bone marrow-derived cells can give rise to ameloblast-like cells [55], which indicates the potential use of non-dental cells for enamel production.

It is anticipated that bioengineered tooth germs will be implanted and grown in the jaw. Support for the feasibility of this approach is provided by recent reports demonstrating that implants placed in the naturally toothless diastema in rodents and in both healed and fresh tooth extraction sockets can accommodate tooth germ maturation in mice and canine jaws. The question that remains is whether human jaws exhibit the same capacity. In addition to identifying the optimized development stage for bioengineered tooth implantation, as well as the optimized implant placement procedure, proper tooth eruption is another important issue and concern. It is currently accepted that the dental follicle plays an important role in the tooth eruption process, as indicated by published reports demonstrating that teeth lacking dental follicles cannot erupt [56]. Teeth can erupt when a developing tooth is replaced by a silicon replica if the dental follicle is retained, but not when the follicle is not retained [57]. Therefore, the creation of bioengineered tooth organs with an associated de novo dental follicle may likely be the optimal design for successful tooth eruption.

Successful whole-tooth regeneration requires the formation of both functional tooth crown and root structures. To date, bioengineered tooth root formation with accompanying functional PDL tissues has proved to be quite challenging, with only a few reports of success [34], indicating that increased effort will be needed to achieve this goal. Finally, potential immune responses to bioengineered human dental implants have yet to be examined and remain virtually unknown at this time. This important feature of biological replacement tooth therapies will require careful attention prior to embarking on clinical trials. Since it appears that human and mouse bone marrow stromal cells do not express costimulatory antigens, DPSCs may similarly represent an immune privileged population and not elicit humoral immune responses [58]. Ideal tooth replacement therapies would use autologous cells harvested from the patient, thereby avoiding potential immunological rejection responses.

\section{Summary and Concluding Remarks}

Dental tissue regeneration provides an attractive alternative to traditional, synthetic tooth restoration therapies. The hope is that patient-specific tissue-derived cell populations can be used to functionally replace integral tooth tissues. The development of such 'test tube teeth' requires precise regulation of the regenerative events in order to achieve proper tooth size and shape, as well as the development of new technologies to facilitate these processes. In the future, it is anticipated that dental tissue development and regeneration will exploit stem cells harvested from dental pulp or use non-DSC sources such as iPS or induced DSCs. The practical use of DSCs for dental tissue engineering applications is at a primitive stage, as current DSC sources are limited to specific developmental times. For example, dental follicle stem cells and SCAP are available only during the wisdom tooth eruption stage in adolescence, while SHED/DPSCs or PDLSCs can be harvested from exfoliated deciduous teeth, from extracted wisdom teeth or from teeth extracted during orthodontic treatment. Fortunately, it has been demonstrated that DSCs can be cryopreserved and used for regenerative purposes at later times. Methods for constructing new tooth tissues include traditional recombination experiments, modified cell pellet cultures, scaffold- 
based tissue engineering, assembly of bioengineered tooth parts and gene therapy-based regeneration strategies. Several existing challenges in regenerative dentistry still need to be overcome, including the need to establish reliable ways to control tooth size, shape and color and to create suitable jaw implantation site environments that enable tooth development in vitro. Finally, methods to control proper functional tooth eruption in adult jaws must also be defined. Based on the current efforts, inter- est and progress, it is tempting to speculate that clinically relevant bioengineered functional tooth therapies for humans may be available in the near future. Although this remains to be seen, it is nevertheless apparent that as our knowledge and understanding of suitable methods for successful dental tissue regeneration continue to increase it can be anticipated that these strategies will lead to the significant benefits offered by biologically based dental tissue replacement therapies.

\section{References}

$\checkmark 1$ Holm-Pedersen P, Schultz-Larsen K, Christiansen N, Avlund K: Tooth loss and subsequent disability and mortality in old age. J Am Geriatr Soc 2008;56:429-435.

-2 Osterberg T, Carlsson GE, Sundh V, Mellstrom D: Number of teeth - a predictor of mortality in 70-year-old subjects. Community Dent Oral Epidemiol 2008;36:258-268.

$\checkmark 3$ Cooper LF: The current and future treatment of edentulism. J Prosthodont 2009;18: 116-122.

4 Tallgren A: The continuing reduction of the residual alveolar ridges in complete denture wearers: a mixed-longitudinal study covering 25 years. 1972. J Prosthet Dent 2003;89: 427-435.

$\checkmark 5$ Kim TI, Jang JH, Kim HW, Knowles JC, Ku Y: Biomimetic approach to dental implants. Curr Pharm Des 2008;14:2201-2211.

6 Kitamura M, Nakashima K, Kowashi Y, Fujii T, Shimauchi H, Sasano T, Furuuchi T, Fukuda M, Noguchi T, Shibutani T, Iwayama Y, Takashiba S, Kurihara H, Ninomiya M, Kido J, Nagata T, Hamachi T, Maeda K, Hara Y, Izumi Y, Hirofuji T, Imai E, Omae M, Watanuki M, Murakami S: Periodontal tissue regeneration using fibroblast growth factor-2: randomized controlled phase II clinical trial. PLoS ONE 2008;3:e2611.

7 Lin C, Dong QS, Wang L, Zhang JR, Wu LA, Liu BL: Dental implants with the periodontium: a new approach for the restoration of missing teeth. Med Hypotheses 2009;72:5861.

8 Cho SW, Kim JY, Cai J, Lee JM, Kim EJ, Lee HA, Yamamoto H, Jung HS: Temporospatial tissue interactions regulating the regeneration of the enamel knot in the developing mouse tooth. Differentiation 2007;75:158165.

9 Mina M, Kollar EJ: The induction of odontogenesis in non-dental mesenchyme combined with early murine mandibular arch epithelium. Arch Oral Biol 1987;32:123-137.
10 Huang X, Xu X, Bringas P, Hung YP, Chai Y: Smad4-Shh-Nfic signaling cascade-mediated epithelial-mesenchymal interaction is crucial in regulating tooth root development. J Bone Miner Res 2009, E-pub ahead of print.

11 Ruch JV: Odontoblast commitment and differentiation. Biochem Cell Biol 1998;76:923938.

12 Zeichner-David M, Oishi K, Su Z, Zakartchenko V, Chen LS, Arzate H, Bringas P Jr: Role of Hertwig's epithelial root sheath cells in tooth root development. Dev Dyn 2003; 228:651-663.

13 Langer R, Tirrell DA: Designing materials for biology and medicine. Nature 2004;428: 487-492.

14 Goldberg M, Farges JC, Lacerda-Pinheiro S, Six N, Jegat N, Decup F, Septier D, Carrouel F, Durand S, Chaussain-Miller C, Denbesten P, Veis A, Poliard A: Inflammatory and immunological aspects of dental pulp repair. Pharmacol Res 2008;58:137-147.

15 Aukhil I, Pettersson E, Suggs C: Guided tissue regeneration: an experimental procedure in beagle dogs. J Periodontol 1986;57:727734.

16 Thomson JA, Itskovitz-Eldor J, Shapiro SS, Waknitz MA, Swiergiel JJ, Marshall VS, Jones JM: Embryonic stem cell lines derived from human blastocysts. Science 1998;282: 1145-1147.

17 Krause DS, Theise ND, Collector MI, Henegariu O, Hwang S, Gardner R, Neutzel S, Sharkis SJ: Multi-organ, multi-lineage engraftment by a single bone marrow-derived stem cell. Cell 2001;105:369-377.

18 Lagasse E, Connors H, Al-Dhalimy M, Reitsma M, Dohse M, Osborne L, Wang X, Finegold M, Weissman IL, Grompe M: Purified hematopoietic stem cells can differentiate into hepatocytes in vivo. Nat Med 2000; 6:1229-1234

19 Gage FH: Mammalian neural stem cells. Science 2000;287:1433-1438.

20 Prockop DJ: Marrow stromal cells as stem cells for nonhematopoietic tissues. Science 1997;276:71-74.
21 Nakagawa M, Koyanagi M, Tanabe K, Takahashi K, Ichisaka T, Aoi T, Okita K, Mochiduki Y, Takizawa N, Yamanaka S: Generation of induced pluripotent stem cells without myc from mouse and human fibroblasts. Nat Biotechnol 2008;26:101-106.

22 Yamanaka S: A fresh look at iPS cells. Cell 2009;137:13-17.

23 Park IH, Daley GQ: Human iPS cell derivation/reprogramming. Curr Protoc Stem Cell Biology 2009; Chapter 4:Unit 4A.1.

24 Sommer CA, Stadtfeld M, Murphy GJ, Hochedlinger K, Kotton DN, Mostoslavsky G: Induced pluripotent stem cell generation using a single lentiviral stem cell cassette. Stem Cells 2009;27:543-549.

25 Okita K, Nakagawa M, Hyenjong H, Ichisaka T, Yamanaka S: Generation of mouse induced pluripotent stem cells without viral vectors. Science 2008;322:949-953.

26 Sveen OB, Hawes RR: Differentiation of new odontoblasts and dentine bridge formation in rat molar teeth after tooth grinding. Arch Oral Biol 1968;13:1399-1409.

27 About I, Bottero MJ, de Denato P, Camps J, Franquin JC, Mitsiadis TA: Human dentin production in vitro. Exp Cell Res 2000;258: 33-41.

28 Gronthos S, Mankani M, Brahim J, Robey PG, Shi S: Postnatal human dental pulp stem cells (DPSCs) in vitro and in vivo. Proc Natl Acad Sci USA 2000;97:13625-13630.

29 Gronthos S, Brahim J, Li W, Fisher LW, Cherman N, Boyde A, DenBesten P, Robey PG, Shi S: Stem cell properties of human dental pulp stem cells. J Dent Res 2002;81:531-535.

-30 Miura M, Gronthos S, Zhao M, Lu B, Fisher LW, Robey PG, Shi S: SHED: stem cells from human exfoliated deciduous teeth. Proc Natl Acad Sci USA 2003;100:5807-5812.

31 Pittenger MF, Mackay AM, Beck SC, Jaiswal RK, Douglas R, Mosca JD, Moorman MA, Simonetti DW, Craig S, Marshak DR: Multilineage potential of adult human mesenchymal stem cells. Science 1999;284:143-147. 
- 32 Satomura K, Krebsbach P, Bianco P, Gehron Robey P: Osteogenic imprinting upstream of marrow stromal cell differentiation. J Cell Biochem 2000;78:391-403.

- 33 d'Aquino R, De Rosa A, Laino G, Caruso F, Guida L, Rullo R, Checchi V, Laino L, Tirino V, Papaccio G: Human dental pulp stem cells: from biology to clinical applications. J Exp Zool B Mol Dev Evol 2009;312B:408415.

-34 Sonoyama W, Liu Y, Fang D, Yamaza T, Seo BM, Zhang C, Liu H, Gronthos S, Wang CY, Shi S, Wang S: Mesenchymal stem cell-mediated functional tooth regeneration in swine. PLoS ONE 2006;1:e79.

- 35 Abe S, Yamaguchi S, Watanabe A, Hamada $\mathrm{K}$, Amagasa T: Hard tissue regeneration capacity of apical pulp derived cells (APDCs) from human tooth with immature apex. Biochem Biophys Res Commun 2008;371:9093.

- 36 Filshie RJ, Zannettino AC, Makrynikola V, Gronthos S, Henniker AJ, Bendall LJ, Gottlieb DJ, Simmons PJ, Bradstock KF: Muc18, a member of the immunoglobulin superfamily, is expressed on bone marrow fibroblasts and a subset of hematological malignancies. Leukemia 1998;12:414-421.

- 37 Seo BM, Miura M, Gronthos S, Bartold PM, Batouli S, Brahim J, Young M, Robey PG, Wang CY, Shi S: Investigation of multipotent postnatal stem cells from human periodontal ligament. Lancet 2004;364:149-155.

- 38 Trubiani O, Orsini G, Zini N, Di Iorio D, Piccirilli M, Piattelli A, Caputi S: Regenerative potential of human periodontal ligament derived stem cells on three-dimensional biomaterials: a morphological report. J Biomed Mater Res A 2008;87:986-993.

39 Shi S, Bartold PM, Miura M, Seo BM, Robey PG, Gronthos S: The efficacy of mesenchymal stem cells to regenerate and repair dental structures. Orthod Craniofac Res 2005;8: 191-199.
40 Morsczeck C, Gotz W, Schierholz J, Zeilhofer F, Kuhn U, Mohl C, Sippel C, Hoffmann KH: Isolation of precursor cells (PCs) from human dental follicle of wisdom teeth. Matrix Biol 2005;24:155-165.

41 Ohshima H, Kenmotsu S, Harada H: Use of the term apical bud to refer to the apical end of the continuously growing tooth. Arch Comp Biol Tooth Enamel 2003;8:45-49.

42 Wang HL, Greenwell H, Fiorellini J, Giannobile W, Offenbacher S, Salkin L, Townsend C, Sheridan P, Genco RJ; Research, Science and Therapy Committee: Periodontal regeneration. J Periodontol 2005;76:1601-1622.

43 Zaman KU, Sugaya T, Kato H: Effect of recombinant human platelet-derived growth factor-BB and bone morphogenetic protein-2 application to demineralized dentin on early periodontal ligament cell response. J Periodontal Res 1999;34:244-250.

44 Taba M Jr, Jin Q, Sugai JV, Giannobile WV: Current concepts in periodontal bioengineering. Orthod Craniofac Res 2005;8:292302.

45 Bashutski JD, Wang HL: Periodontal and endodontic regeneration. J Endod 2009;35: 321-328.

46 Thesleff I: Differentiation of odontogenic tissues in organ culture. Scand J Dent Res 1976;84:353-356.

47 Peterka M, Mandys V, Peterková R: A modification of tooth germ cultivation in vitro and in ovo. Cytotechnology 1991;7:49-53.

48 Isogawa N, Terashima T, Nakano Y, Kindaichi J, Takagi Y, Takano Y: The induction of enamel and dentin complexes by subcutaneous implantation of reconstructed human and murine tooth germ elements. Arch Histol Cytol 2004;67:65-77.

49 Young CS, Terada S, Vacanti JP, Honda M, Bartlett JD, Yelick PC: Tissue engineering of complex tooth structures on biodegradable polymer scaffolds. J Dent Res 2002;81:695700 .
50 Hu B, Nadiri A, Kuchler-Bopp S, PerrinSchmitt F, Peters H, Lesot H: Tissue engineering of tooth crown, root, and periodontium. Tissue Eng 2006;12:2069-2075.

51 Komine A, Suenaga M, Nakao K, Tsuji T, Tomooka Y: Tooth regeneration from newly established cell lines from a molar tooth germ epithelium. Biochem Biophys Res Commun 2007;355:758-763.

52 Abukawa H, Zhang W, Young CS, Asrican R, Vacanti JP, Kaban LB, Troulis MJ, Yelick PC: Reconstructing mandibular defects using autologous tissue-engineered tooth and bone constructs. J Oral Maxillofac Surg 2009;67:335-347.

-53 Kuo TF, Huang AT, Chang HH, Lin FH, Chen ST, Chen RS, Chou $\mathrm{CH}$, Lin $\mathrm{HC}$, Chiang H, Chen MH: Regeneration of dentin-pulp complex with cementum and periodontal ligament formation using dental bud cells in gelatin-chondroitin-hyaluronan tricopolymer scaffold in swine. J Biomed Mater Res A 2008;86:1062-1068.

54 Ohazama A, Modino SA, Miletich I, Sharpe PT: Stem-cell-based tissue engineering of murine teeth. J Dent Res 2004;83:518-522.

$55 \mathrm{Hu}$ B, Unda F, Bopp-Kuchler S, Jimenez L, Wang XJ, Haikel Y, Wang SL, Lesot H: Bone marrow cells can give rise to ameloblast-like cells. J Dent Res 2006;85:416-421.

56 Larson EK, Cahill DR, Gorski JP, Marks SC Jr: The effect of removing the true dental follicle on premolar eruption in the dog. Arch Oral Biol 1994;39:271-275.

57 Cahill DR, Marks SC Jr: Tooth eruption: evidence for the central role of the dental follicle. J Oral Pathol 1980;9:189-200.

58 Pierdomenico L, Bonsi L, Calvitti M, Rondelli D, Arpinati M, Chirumbolo G, Becchetti E, Marchionni C, Alviano F, Fossati V, Staffolani N, Franchina M, Grossi A, Bagnara GP: Multipotent mesenchymal stem cells with immunosuppressive activity can be easily isolated from dental pulp. Transplantation 2005;80:836-842. 